\title{
Ambiente de Prática de Enfermagem em contexto hospitalar: Revisão integrativa
}

\author{
Sara Anunciada ${ }^{1}$ e Pedro Lucas ${ }^{2}$ \\ ${ }^{1} \mathrm{MScN}$, Escola Superior de Enfermagem de Lisboa. ACES Lisboa Central, Lisboa, Portugal \\ | saraanunciada@gmail.com | https://orcid.org/0000-0002-6062-4961 \\ ${ }^{2} \mathrm{PhD}, \mathrm{MSc}, \mathrm{MScN}, \mathrm{RN}$, Nursing Research, Innovation and Development Centre of \\ Lisbon (CIDNUR). Nursing School of Lisbon, Lisbon, Portugal | prlucas@esel.pt | \\ https://orcid.org/0000-0002-2560-7306
}

Resumo: O contexto de trabalho onde os enfermeiros prestam cuidados desempenha um papel relevante nos resultados para o cliente e para os enfermeiros e respetivas equipas. As diferentes características do Ambiente de Prática em Enfermagem (APE) são importantes na construção de estratégias de gestão para a promoção de APE favoráveis, que por consequentemente contribuem para a melhoria contínua da Qualidade dos Cuidados em Enfermagem. Objetivos: examinar a evidência científica acerca do APE em contexto hospitalar. Métodos: Revisão integrativa, com 6 etapas: 1) identificação da pergunta de pesquisa; 2) definição das palavras-chave; 3) definição das informações a serem extraídas dos estudos selecionados; 4) avaliação dos estudos incluídos na revisão; 5) interpretação dos resultados; 6) apresentação da revisão/síntese do conhecimento. Os estudos selecionados foram no período entre 2015 e 2020. Resultados: Foram incluídos 26 artigos; a grande maioria relatou achados do ambiente de prática de enfermagem e resultados para os enfermeiros, para os enfermeiros gerentes, para os clientes e para a eficácia das organizações, em contexto hospitalar. Conclusões: Melhorar o ambiente de prática de enfermagem tem consequências na qualidade dos cuidados de enfermagem, com aumento de resultados para a enfermagem, os clientes e o contexto hospitalar.

Palavras-chave: Ambiente de Trabalho; Enfermagem; Hospital; Revisão.

\section{Nursing Practice Environment in a Hospital Context: Integrative Review}

Abstract: The work context where nurses provide care plays an important role in the results for the client and for the nurses and their teams. The different characteristics of the Nursing Practice Environment are important in the construction of management strategies for the promotion of favorable work environment, which consequently contribute to the continuous improvement of the Quality of Nursing Care. Objectives: to examine the scientific evidence about the EPA in a hospital context. Methods: Integrative review, with 6 steps: 1) identification of the research question; 2) definition of keywords; 3) definition of the information to be extracted from the selected studies; 4 ) evaluation of the studies included in the review; 5 ) interpretation of results; 6) presentation of the review / synthesis of knowledge. The selected studies were in the period between 2015 and 2020. Results: 26 articles were included; the vast majority reported findings of the nursing practice environment and results for nurses, for nurse managers, for clients and for the effectiveness of organizations, in the hospital context. Conclusions: Improving the nursing practice environment has consequences for the quality of nursing care, with an increase in results for nursing, clients and the hospital context.

Keywords: Nursing; Hospital; Review; Work Environment.

\section{Introdução}

O Ambiente de Prática de Enfermagem (APE) é fundamental para o sucesso dos sistemas de saúde (Almeida, Nascimento, Lucas, Jesus \& Araújo, 2020) e está relacionado com a qualidade dos cuidados de enfermagem (QCE), com a satisfação profissional, com a segurança do cliente e com a efetividade dos cuidados para os clientes e para a eficiência das organizações (Carvalho \& Lucas, 2020; De Sul \& Lucas, 2020; Lake, 2002; Lucas \& Nunes, 2020). Segundo Lake (2002), APE é definido como as características organizacionais de um contexto de trabalho que facilitam ou constrangem a prática profissional dos enfermeiros. 
Promover a qualidade dos cuidados que os enfermeiros prestam e, portanto, contribuir para a melhoria dos contextos das práticas clínicas é um fator fundamental do APE. A QCE é um elemento essencial na profissão e refere-se, entre outros aspetos, à relação direta entre o cliente e o enfermeiro, dependendo de muitos fatores, principalmente do APE (Lucas \& Nunes, 2020, Tomaszewska, Kłos \& Majchrowicz, 2017).

Um APE favorável leva à melhoria dos resultados dos clientes, sendo um fator essencial para o aumento da satisfação dos enfermeiros (Lake, 2002; Leone et al., 2015) e para se manterem equipas com dotações seguras, retendo os enfermeiros (De Sul \& Lucas, 2020; Lucas \& Nunes, 2020). APE favorável é caracterizado pela adequação de recursos humanos e materiais, participação ativa dos enfermeiros na governação das organizações, qualidade do atendimento e de prestação de cuidados de enfermagem, e boas relações entre os diferentes grupos profissionais dos serviços de saúde (Almeida et al., 2020; Lake, 2002). De acordo com a evidência científica das últimas décadas, estes APE favoráveis têm impactos significativos nos níveis de qualidade e segurança dos cuidados ao cliente, bem-estar dos profissionais de saúde, qualidade e produtividade, e eficácia dos serviços, organizações e sistemas de saúde (Almeida et al., 2020).

Por outro lado, APE pobres, com falta de apoio da gestão, fraca liderança e má relação enfermeiro-médico estão associados a: diminuição da QCE; eventos adversos nos clientes (Poghosyan et al., 2015), como erros; aumento da mortalidade e complicações; reinternamentos por complicações; aumento dos custos com os cuidados de saúde; prestação ineficaz de cuidados, conflitos e stress entre os profissionais de saúde (Tomaszewska et al., 2017); insatisfação profissional e aumento da rotatividade dos enfermeiros (Lucas \& Nunes, 2020). Todos estes aspetos contribuem fortemente para a insatisfação dos clientes com os cuidados que lhes são prestados.

Um APE seguro caracteriza-se por boas relações profissionais entre os seus membros, apoio da gestão aos profissionais e horários de trabalho equilibrados (Alves \& Guirardello, 2016; Copanitsanou, Fotos \& Brokalaki, 2017; Lucas \& Nunes, 2020). Caracteriza-se também por adequação entre a carga de trabalho e as competências dos enfermeiros, tempo para dar resposta às necessidades dos clientes, autonomia profissional, recursos adequados e oportunidades de progressão profissional (Alves \& Guirardello, 2016; Copanitsanou et al., 2017; Lucas \& Nunes, 2020).

Os enfermeiros gestores desempenham um papel fundamental na criação de um APE favorável, positivo (Alves \& Guirardello, 2016), e na promoção de uma prestação de cuidados de qualidade (Carvalho \& Lucas, 2020; Lucas \& Nunes, 2020). Podem ainda proporcionar as ferramentas necessárias para o desenvolvimento profissional dos enfermeiros e de futuros gestores (Gea-Caballero et al., 2018). A liderança em enfermagem desempenha um papel central nos cuidados de qualidade ao cliente, o qual envolve quatro atividades fundamentais: facilitar a comunicação contínua eficaz; fortalecimento das relações intra e interprofissionais; construção e manutenção de equipas e envolvimento dos pares (Carvalho \& Lucas, 2020). A liderança influencia o APE (Lucas \& Nunes, 2020; Weber, Ward \& Walsh, 2015) e a QCE (Carvalho \& Lucas, 2020). Os enfermeiros, enquanto líderes, são fundamentais para melhorarem a comunicação com e entre a equipa para alcançarem objetivos, tendo como finalidade a qualidade dos cuidados, a segurança do cliente e a inovação em saúde (Carvalho \& Lucas, 2020; Nunes \& Gaspar, 2016).

Sem competências e conhecimentos adequados, torna-se difícil para os líderes em enfermagem manterem um ambiente de prática favorável (Alves \& Guirardello, 2016; Lucas \& Nunes, 2020). O enfermeiro gestor é um motor de mudança no caminho para a excelência, organizando os recursos existentes e criando um ambiente seguro nos cuidados de enfermagem (Lucas \& Nunes, 2020).

Assim, visto que um APE favorável é essencial para a implementação de qualquer programa de saúde em contexto hospitalar a promoção desses ambientes é fundamental, sendo um serviço público aos cidadãos, aos profissionais e às organizações de saúde. 


\section{Material e Métodos}

Esta revisão seguiu as seguintes etapas: identificação da pergunta de pesquisa; definição das palavras-chave para pesquisa na literatura; definição das informações a serem extraídas dos estudos selecionados/categorização dos estudos; avaliação dos estudos incluídos na revisão integrativa; interpretação dos resultados; apresentação da revisão/síntese do conhecimento (Whittemore \& Knafl, 2005).

O objetivo da revisão é examinar a evidência científica acerca do APE em contexto hospitalar, orientada pela pergunta: Quais as características do APE no contexto hospitalar?

A pesquisa foi realizada nas bases de dados CINAHL, MEDLINE, Scopus, LILACS, Cochrane Database of Systematic Reviews, Repositórios Científicos de Acesso Aberto de Portugal (RCAAP), com uma análise das palavras-chave identificadas nos Descritores em Ciências da Saúde (DeCS) e no Medical Subjet Heading (Mesh). O operador booleano usado foi AND e OR. Os termos utilizados foram: Nursing Practice Environment OR working environment $O R$ work environment AND hospital. Foram aplicados como limitadores, artigos disponíveis em texto integral.

$\mathrm{Na}$ inclusão dos estudos foram considerados artigos que abordassem o tema do APE, com enfermeiros de todas as faixas etárias, de todas as áreas de especialidade e desenvolvimento profissional, que exercem funções em contexto hospitalar, estudos primários e secundários. Artigos disponíveis em texto integral e publicados em inglês, português ou castelhano, no período temporal entre 2015 e 2020.

Os dados extraídos dos artigos incluídos na revisão foram alinhados com o objetivo e questão de pesquisa. Elaborou-se uma tabela de extração que se encontra organizada pelos seguintes dados: Autor(es), Ano de publicação; Título; País; Objetivos; Desenho do estudo; População em estudo/Tamanho da amostra/Participantes; Contexto; Principais resultados.

\section{Resultados}

Após a remoção dos artigos duplicados, identificaram-se 515 artigos para a seleção da revisão. Um total de 53 artigos cumpria os critérios de inclusão com base na verificação dos títulos e dos resumos. Obtidos os artigos de texto completo foram então lidos e examinados e 26 cumpriram os critérios de inclusão. Ao fim, esta revisão da literatura correspondeu à resposta de 33.384 enfermeiros e 24.258 clientes do contexto hospitalar.

Apenas 3 dos 6 continentes foram representados neste estudo. A Europa apresentou o maior número de publicações, com 6 estudos. A América foi representada pelo Brasil com 4 publicações; e a Ásia (Omã e China) com 5 estudos.

O método de investigação quantitativo foi adotado em todos os estudos. Foram encontradas e incluídas neste estudo duas revisões scoping, três revisões sistemáticas da literatura e uma meta-análise, totalizando 173 estudos. Na qualidade dos estudos considerou-se os 7 níveis de evidência, agrupados segundo Melnyk \& Fineout-Overholt, (2011), evidências fortes, moderadas e fracas. Foram respeitados os aspetos éticos da pesquisa, bem como os conceitos e definições presentes nos estudos selecionados.

A Fig. 1., especifica os resultados das etapas da análise, seguindo o modelo PRISMA Flow Diagram (Mozer et al., 2009). 


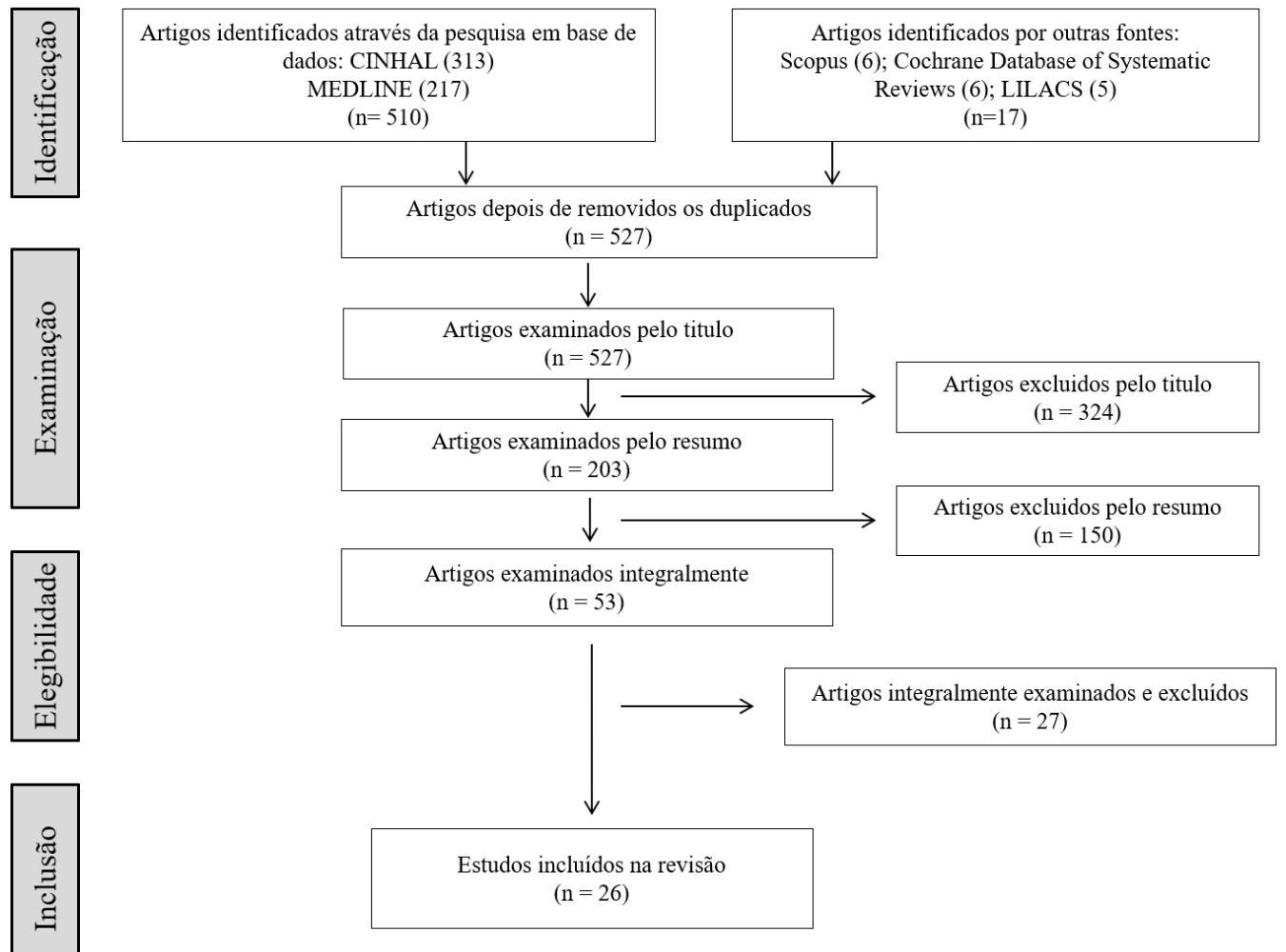

Fig. 1. Diagrama de PRISMA referente ao processo de seleção dos estudos.

\section{Discussão}

As características do APE assumem-se como importantes fatores nas organizações de saúde, podendo afetar a qualidade e a segurança dos cuidados. A presença dos atributos organizacionais, sobretudo a autonomia, o controlo sobre o ambiente e a colaboração entre os profissionais de saúde, faz com que o APE seja benéfico ao desenvolvimento dos cuidados, bem como a contribuição para melhores outcomes para o cliente, profissionais de saúde e para a própria instituição, resultando na QCE prestados (Aiken \& Patrician, 2000; Duffield et al., 2011; Kirwan, Matthews, \& Scott, 2013).

Os estudos que foram realizados em países como Bélgica, Inglaterra, Finlândia, Irlanda, Espanha, Suíça, Brasil e China demonstraram que um ambiente favorável no local de trabalho favorece uma melhor qualidade de prática de enfermagem, com obtenção de melhores resultados (Aiken et al., 2017; Alves \& Guirardello, 2016; Bai, 2016; Guirardello, 2017; Liu et al., 2018; Zhu, Zheng, Liu, \& You, 2019) e, consequentemente, maior satisfação dos clientes (Chen, Ramalhal, \& Lucas, 2019).

Assim, um APE "positivo contribui para a satisfação dos enfermeiros e para a retenção dos mesmos nas organizações, conseguindo assim uma maior qualidade dos cuidados de enfermagem, redução de custos para a organização hospitalar, maiores resultados nos clientes e nas equipas de enfermeiros" (Chen et al., 2019, p. 38).

Copanitsanou et al., (2017) e Chen et al., (2019) referem que um APE seguro se caracteriza por boas relações profissionais, por uma boa gestão de apoio, rácios adequados, tempo adequado para prestar cuidados, de acordo com as necessidades dos clientes, autonomia e desenvolvimento profissional. 
O APE tem impacto nos resultados dos cliente e dos enfermeiros e os ambientes onde os enfermeiros possuem autonomia, controlo sobre o ambiente e boas relações com a equipa médica resultam em menores níveis de burnout (AL Ma'mari, Sharour, \& Al Omari, 2020; Chen et al., 2019; Guirardello, 2017; Liu et al., 2018; Nogueira et al., 2018; Xian, Zhai, Xiong, \& Han, 2020), maior satisfação profissional (Almeida et al., 2020; Chen et al., 2019; De Sul \& Lucas, 2020; Dorigan \& Guirardello, 2017; Ventura, Ramalhal, \& Lucas, 2019), menor intenção de deixar o emprego (Alves \& Guirardello, 2016; De Sul \& Lucas, 2020; Leone et al., 2015; Lucas \& Nunes, 2020; Xian et al., 2020) e melhores resultados para os clientes no que diz respeito à qualidade dos cuidados (Aiken et al., 2017; Almeida et al., 2020; Alves \& Guirardello, 2016; Guirardello, 2017; Lucas \& Nunes, 2020; Zhu et al., 2019) e segurança dos clientes (Aiken et al., 2017; Almeida et al., 2020; Liu et al., 2018; Lucas \& Nunes, 2020).

Na mesma linha de pensamento, Lake et al., (2019) concluiram que melhores APE estão associados a menores probabilidades de resultados negativos, menor insatisfação no trabalho e menor mortalidade dos clientes.

Lake (2007) refere que o APE deve ser o foco principal de preocupação dos sistemas de saúde para evitar crises em duas áreas: na escassez de enfermeiros e na segurança dos clientes. Assim, monitorizar o APE e minimizar as suas lacunas, permitirá a implementação de processos de melhoria contínua, melhorando por sua vez os resultados relacionados com a saúde. Neste contexto, o uso de instrumentos destinados a avaliar os APE é fundamental para melhorar a QCE (Aiken, Clarke, Sloane, Lake, \& Cheney, 2008; Fuentelsaz-Gallego, Moreno-Casbas, \& González-María, 2013; Laschinger, Zhu, \& Read, 2016; Neves et al., 2018).

Os artigos selecionados utilizaram os seguintes instrumentos de medida: Practice Environment Scale- Nursing Work Index (PES-NWI), Nursing Work Index-Revised (NWIR), Safety Attitudes Questionnaire Short Form 2006 - (SAQ), Essentials of Magnetism II (EOM II), Overall Job Satisfaction (OJS), Quality of Care (QC), Maslach Burnout InventoryHuman Services Survey (MBI-HSS), Maslach Burnout Inventory (MBI) e Anticipated Turnover Scale (ATS), The Multifactor Leadership Questionnaire (MLQ), The Psychological Empowerment Scale, The Three-Component Model of Employee Commitment, Nursing Home Survey on Patient Safety Culture (NHSPSC), American Association of Critical-Care Nurses (AACN) Healthy Work Environment Assessment Tool, Authentic Leadership Questionnaire, Areas of Worklife Scale, Occupational Coping SelfEfficacy, General Health Questionnaire, Leiden Quality of Work Questionnaire (LQWQ), Leiden Quality of Work Questionnaire for Nurses (LQWQ-N). A PES-NWI continua a ser 0 instrumento de medida mais utilizado para avaliação do APE (Lake, 2007).

A avaliação do APE pode parecer mais essencial para o exercício das funções dos enfermeiros gestores, mas é igualmente relevante para os enfermeiros prestadores de cuidados. Os enfermeiros prestadores de cuidados diretos estão cada vez mais envolvidos na tomada de decisões organizacionais e, assim, têm a oportunidade de projetar e implementar soluções para as questões de interesse da sua unidade. Melhorar o APE permitirá aos enfermeiros dedicarem mais tempo ao tratamento direto do cliente, contribuindo decisivamente para a qualidade dos cuidados que the são prestados (Adriaenssens et al., 2017). Assim, os enfermeiros gestores devem criar APE que envolvam os enfermeiros nas decisões que influenciam os cuidados ao cliente e o ambiente de trabalho (Mrayyan, 2019).

Os enfermeiros gestores têm um papel fundamental na organização dos cuidados de saúde, tendo no entanto que lidar com questões e problemas relacionados com o trabalho, num ambiente de cuidados de saúde em constante mudança e desafiador (Adriaenssens et al., 2017). Estes, através da liderança e gestão, têm uma influência significativa na qualidade e segurança dos cuidados e no bem-estar dos enfermeiros (Adriaenssens et al., 2017). Laschinger, Borgogni, Consiglio \& Read (2015), referem que o comportamento de liderança autêntica dos gestores de enfermagem, como a autoconsciência e transparência, comportamento ético-moral e apoio nos processos, desempenham um papel importante na criação de APE favoráveis. 
Os enfermeiros gestores podem melhorar o APE, praticando uma liderança adequada e estratégias de empoderamento, incluindo uma maior participação da equipa de enfermagem no processo de tomada de decisão, levando assim a cuidados de enfermagem de alta qualidade, que necessariamente conduzirão a uma maior segurança do cliente (Asiri, Rohrer, Al-Surimi, Da'ar, \& Ahmed, 2016).

A colaboração com médicos e outros profissionais foi positivamente relacionada com a satisfação no trabalho. A cooperação solidária, a boa comunicação e a tomada de decisões interdisciplinares conjuntas influenciam positivamente o grau de satisfação e realização das equipas de enfermagem com consequências concretas (Adriaenssens et al., 2017). Índices de realização mais elevados nas equipas de enfermagem estão associados a menos quedas de clientes e a um melhor controlo da dor em relação às úlceras por pressão (Stalpers et al., 2015). Acresce que se verifica também que níveis mais altos de experiência e educação, assim como relações de colaboração harmoniosa entre profissionais, têm efeitos favoráveis nos resultados de quedas e úlceras de pressão nos clientes (Stalpers et al., 2015).

Os enfermeiros com pouca autonomia têm efeitos negativos na satisfação no trabalho $e$ na intenção de saída Mrayyan (2019). Ko et al., (2018) concluíram que existe uma necessidade de melhorar a autonomia do trabalho dos enfermeiros, pois tem implicações no APE. Assim, os enfermeiros gestores devem monitorizar a autonomia do trabalho dos enfermeiros e as suas perceções sobre a política organizacional (Ko et al., 2018) e avaliar precocemente a intenção de saída através de instrumentos de medida, evitando este fenómeno, contribuindo para a melhoria dos APE (De Sul \& Lucas, 2020). Os enfermeiros gestores podem desenvolver a formação em serviço e implementar métodos de trabalho que promovam e melhoram a autonomia do trabalho, criando assim APE favoráveis, que permitam aos enfermeiros participarem na tomada de decisões (Ko et al., 2018). Defendem ainda que, estes esforços favorecem a QCE, garantindo a segurança do cliente (Ko et al., 2018).

Titlestad, Haugstvedt, Igland, \& Graue (2018), sugerem que as linhas orientadoras de atuação dos serviços de saúde contribuem para garantir a aplicação de novos conhecimentos na prática, reduzindo efeitos indesejáveis, variações de práticas e melhorando a segurança e a qualidade. Estes autores referem ainda que os enfermeiros com formação avançada e utilizadores de linhas orientadoras, ou guidelines, têm perceções mais positivas sobre áreas chave da segurança do cliente (Titlestad et al., 2018). Quanto maior a formação dos enfermeiros, melhor conhecimento e capacidades estes possuem para lidar com diferentes situações relativamente ao cuidado do cliente e ao trabalho em equipa e equipa multidisciplinar e maior é a capacidade para adotar uma abordagem mais abrangente para a enfermagem e para a segurança do cliente (Titlestad et al., 2018). Deve então o enfermeiro gestor incentivar a formação formal da sua equipa como forma de promover cuidados de alta qualidade e segurança. Também Weber, Ward, e Walsh (2015), defendem que os enfermeiros gestores têm um papel preponderante dentro da organização e na prestação de um atendimento de qualidade, que é o de possibilitar e proporcionar as ferramentas necessárias, para o desenvolvimento profissional dos enfermeiros e futuros gestores.

A formação em contexto profissional e a formação base das escolas de enfermagem e nos cursos de pós-graduação/especializações devem incluir programas educativos com conteúdos sobre as caraterísticas organizacionais, o APE e a sua relação com a saúde e os resultados do trabalho, preparando assim futuros enfermeiros para reconhecerem e promoverem ambientes de trabalho saudáveis (Lake et al., 2019; Mrayyan, 2019). Lake et al., (2019) referem que os enfermeiros, na prestação de cuidados, também merecem educação sobre as características de ambientes de trabalho saudáveis (Ibrahim et al., 2014). Weber et al., (2015) referem que um bom relacionamento entre os enfermeiros gestores e os enfermeiros é a base para o empoderamento dos enfermeiros, resultando em baixos níveis de burnout e melhor satisfação profissional.

Os líderes e administradores de enfermagem devem promover características organizacionais e APE de Magnet Hospitals para melhorar a segurança dos clientes e a qualidade dos cuidados (Wei, Sewell, Woody, \& Rose, 2018). 
Os hospitais que possuem estas características atraem enfermeiros por terem características organizacionais hospitalares positivas e APE saudáveis, que por sua vez, possuem resultados positivos nos enfermeiros, nos clientes e nas organizações (Er \& Sökmen, 2018).

A avaliação dos serviços tem como objetivo ajudar os gestores de enfermagem a desenvolverem APE favoráveis para a prestação de cuidados de enfermagem de alta qualidade. Estas avaliações auxiliam os gestores a entender como os enfermeiros percecionam o seu APE e como isso influencia o seu trabalho e os cuidados que prestam. Os resultados destas avaliações devem ser utilizados pelos gestores de enfermagem para alcançarem resultados positivos para os clientes, satisfação dos enfermeiros, prestação de cuidados de qualidade, segurança dos clientes e diminuição da intenção de saída dos enfermeiros (Er \& Sökmen, 2018).

Assim, os gestores devem avaliar os seus APE através de escalas existentes, de modo a para identificarem áreas de fraqueza nos seus ambientes de prática de enfermagem (Lake et al., 2019).

\section{Conclusão}

O APE, decorrente das características da organização onde se desenvolve a prestação de cuidados, é determinante para a QCE prestados aos clientes.

Verifica-se que a QCE, sobretudo em contexto hospitalar, tem sido o foco de estudos nacionais e internacionais, os quais revelam que determinadas características do APE favorecem a prática dos enfermeiros, proporcionando maior satisfação profissional, contribuindo de forma positiva para a segurança dos clientes. Os estudos evidenciam ainda que os APE com maior autonomia, maior controlo e maior suporte organizacional, bem como ambientes com boas relações da equipa multidisciplinar, resultam em avaliações mais positivas por parte dos enfermeiros acerca da QCE.

Um APE favorável é um fator decisivo na satisfação, manutenção e resultados dos clientes. Assim, para compreender como a estrutura, a cultura e a organização dos hospitais afetam os resultados dos clientes e da enfermagem, a literatura fornece ferramentas que foram desenvolvidas para avaliar a presença de determinadas características ambientais da prática profissional de enfermagem.

Os gestores em enfermagem e as organizações hospitalares devem encarar o desafio da melhoria do APE como um investimento que, inevitavelmente, se refletirá na melhoria dos resultados aos clientes, na performance profissional e, sobretudo, na qualidade dos cuidados prestados, independentemente do contexto onde esse investimento ocorra.

Fica explícita a relação profunda que os enfermeiros estabelecem com o seu APE e a influência que o enfermeiro gestor pode deter sobre os mesmos, dando origem aos mais variados outcomes. Consequentemente, o enfermeiro gestor, através da sua gestão, influencia favoravelmente o APE, considerando que promove um aumento na QCE, que por sua vez vai promover outcomes positivos para os clientes e enfermeiros, aumentando a eficácia das organizações, melhorando as organizações privadas e o Serviço Nacional de Saúde.

Assim, melhorar o APE tem consequências na QCE, com aumento de resultados para a enfermagem, para os clientes e para o contexto hospitalar. 


\section{Referências}

Adriaenssens, J., Hamelink, A., \& Van Bogaert, P. (2017). Predictors of occupational stress and well-being in First-Line Nurse Managers: A cross-sectional survey study. International Journal of Nursing Studies, 73, 85-92. Doi:10.1016/j.ijnurstu.2017.05.007.

Aiken, L. H., Clarke, S. P., Sloane, D. M., Lake, E. T., \& Cheney, T. (2008). Effects of Hospital Care Environment on Patient Mortality and Nurse Outcomes. Journal of Nurse Administration, 38(5), 223-229. Doi:10.1097/01.NNA.0000312773.42352.d7.

Aiken, L. H., \& Patrician, P. A. (2000). Measuring Organizational Traits of Hospitals: The Revised Nursing Work Index. Nursing Research, 49(3), 146-153.

Aiken, L. H., Sloane, D., Griffiths, P., Rafferty, A. M., Bruyneel, L., McHugh, M., ... Van Achterberg, T. (2017). Nursing skill mix in European hospitals: Cross-sectional study of the association with mortality, patient ratings, and quality of care. BMJ Quality and Safety, 26(7), 559-568. Doi:10.1136/bmjqs-2016-005567.

AL Ma'mari, Q., Sharour, L. A., \& Al Omari, O. (2020). Fatigue, burnout, work environment, workload and perceived patient safety culture among critical care nurses. British Journal of Nursing, 29(1), 28-34. Doi:10.12968/bjon.2020.29.1.28.

Almeida, S., Nascimento, A., Lucas, P. B., Jesus, E., \& Araújo, B. (2020). RN4CAST Study in Portugal: Validation of the Portuguese Version of the Practice Environment Scale of the Nursing Work Index. Aquichan, 20(3), e2038. Doi:10.5294/aqui.2020.20.3.8.

Alves, D. F. S., \& Guirardello, E. B. (2016). Nursing work environment, patient safety and quality of care in pediatric hospital. Revista Gaucha de Enfermagem, 37(2), e58817. Doi:10.1590/19831447.2016.02.58817.

Asiri, S. A., Rohrer, W. W., Al-Surimi, K., Da'ar, O. O., \& Ahmed, A. (2016). The association of leadership styles and empowerment with nurses' organizational commitment in an acute health care setting: A cross-sectional study. BMC Nursing, 15(1), 1-10. Doi:10.1186/s12912016-0161-7.

Bai, J. (2016). Does job satisfaction mediate the relationship between healthy work environment and care quality? Nursing in Critical Care, 21(1), 18-27. Doi:10.1111/nicc.12122.

Carvalho, M. C., \& Lucas, P. R. (2020). The effectiveness of the clinical nurse leader practice systematic review. Millenium, 2(11), 57-64. Doi:10.29352/mill0211.06.00274.

Chen, J., Ramalhal, T., \& Lucas, P. (2019). Ambiente de Prática de Enfermagem e a Satisfação dos Enfermeiros em Contexto Hospitalar - Uma Revisão Scoping. Pensar Enfermagem, 23(2), 29-42.

Copanitsanou, P., Fotos, N., \& Brokalaki, H. (2017). Effects of work environment on patient and nurse outcomes. British Journal of Nursing, 26(3), 172-176. Doi:10.12968/bjon.2017.26.3.172.

De Sul, S. I. R., \& Lucas, P. R. M. B. (2020). Translation and validation of the anticipated turnover scale for the Portuguese cultural context. Nursing Open, 00, 1-7. Doi:10.1002/nop2.521.

Dorigan, G. H., \& Guirardello, E. B. (2017). Nursing practice environment, satisfaction and safety climate: The nurses' perception. Acta Paulista de Enfermagem, 30(2), 129-135. Doi:10.1590/1982-0194201700021.

Duffield, C., Diers, D., O’Brien-Pallas, L., Aisbett, C., Roche, M., King, M., \& Aisbett, K. (2011) Nursing staffing, nursing workload, the work environment and patient outcomes. Applied Nursing Research, 24(4), 244-255. Doi:10.1016/j.apnr.2009.12.004.

Fuentelsaz-Gallego, C., Moreno-Casbas, M. T., \& González-María, E. (2013). Validation of the Spanish version of the questionnaire Practice Environment Scale of the Nursing Work Index. International Journal of Nursing Studies, 50(2), 274-280. Doi:10.1016/j.ijnurstu.2012.08.001.

Gea-Caballero, V., Castro-Sánchez, E., Júarez-Vela, R., Díaz-Herrera, M. Á., Miguel-Montoya, I. \& Martínez-Riera, J. R. (2018). Elementos esenciales de los entornos profesionales enfermeros en Atención Primaria y su influencia en la calidad del cuidado. Enfermería Clínica, 28(1), 2735. Doi:10.1016/j.enfcle.2017.07.008. 
Guirardello, E. B. (2017). Impact of critical care environment on burnout, perceived quality of care and safety attitude of the nursing team. Revista Latino-Americana de Enfermagem, 25(0), e2884. Doi:0.1590/1518-8345.1472.2884.

Kirwan, M., Matthews, A., \& Scott, P. A. (2013). The impact of the work environment of nurses on patient safety outcomes: A multi-level modelling approach. International Journal of Nursing Studies, 50(2), 253-263. Doi:10.1016/j.jnurstu.2012.08.020.

Lake, E. T. (2002). Development of the practice environment scale of the nursing work index. Research in Nursing and Health, 25 (3), 176-188. Doi:10.1002/nur.10032

Lake, E. T. (2007). The Nursing Practice Environment:Measurement and Evidence. Medical Care Research and Review, 64(2), 104S-122S. Doi:10.1177/1077558707299253.

Lake, E. T., Sanders, J., Duan, R., Riman, K. A., Schoenauer, K. M., \& Chen, Y. (2019). A MetaAnalysis of the Associations between the Nurse Work Environment in Hospitals and 4 Sets of Outcomes. Medical Care, 57(5), 353-361. Doi:10.1097/MLR.0000000000001109.

Laschinger, H. K. S., Borgogni, L., Consiglio, C., \& Read, E. (2015). The effects of authentic leadership, six areas of worklife, and occupational coping self-efficacy on new graduate nurses' burnout and mental health: A cross-sectional study. International Journal of Nursing Studies, 52(6), 1080-1089. Doi:10.1016/j.jjnurstu.2015.03.002.

Laschinger, H. K. S., Zhu, J., \& Read, E. (2016). New nurses' perceptions of professional practice behaviours, quality of care, job satisfaction and career retention. Journal of Nursing Management, 24(5), 656-665. Doi:10.1111/jonm.12370.

Leone, C., Bruyneel, L., Anderson, J. E., Murrells, T., Dussault, G., Jesus, É. H., ... Rafferty, A. M. (2015). Work environment issues and intention-to-leave in Portuguese nurses: A crosssectional study. Health Policy, 119(12), 1584-1592. Doi:10.1016/j.healthpol.2015.09.006.

Liu, X., Zheng, J., Liu, K., Baggs, J. G., Liu, J., Wu, Y., \& You, L. (2018). Hospital nursing organizational factors, nursing care left undone, and nurse burnout as predictors of patient safety: A structural equation modeling analysis. International Journal of Nursing Studies, 86, 82-89. Doi:10.1016/j.ijnurstu.2018.05.005.

Lucas, P. R. M. B., \& Nunes, E. M. G. T. (2020). Nursing practice environment in Primary Health Care: a scoping review. Revista Brasileira de Enfermagem, 73(6), e20190479. Doi:10.1590/0034-7167-2019-0479.

Melnyk, B. \& Fineout-Overholt, E. (2011). Evidence-based practice in nursing \& healthcare: A guide to best practice. Philadelphia: Lippincott Williams \& Wilkins.

Moher, D., Liberati, A., Tetzlaff, J., Atman, D., \& The PRISMA Group. (2009). Preferred reporting items for systematic reviews and meta-analyses: the PRISMA statement. PloS medicine, 6 (7), e1000097. Doi:10.1371/journal.pmed.1000097.

Mrayyan, M. T. (2019). Nurses' views on hospital organizational characteristics. Nursing Forum, 54(4), 650-660. Doi:10.1111/nuf.12390.

Neves, T. M. A., Parreira, P. M. S. D., Graveto, J. M. G. N., Rodrigues, V. J. L., \& Marôco, J. P. D. (2018). Practice environment scale of the nursing work index: Portuguese version and psychometric properties. Journal of Nursing Management, 26(7), 833-841. Doi:10.1111/jonm.12606.

Nogueira, L. S., Sousa, R. M. C., Guedes, E. S., Turrini, R. N. T., Cruz, D. A. L. M., \& Santos, M. A. (2018). Burnout and nursing work environment in public health institutions. Revista Brasileira de Enfermagem, 71(2), 358-365. Doi:10.1590/0034-7167-2016-0524.

Nunes, E. M. G. T. \& Gaspar, M. F. M. (2016) A liderança em enfermagem e a satisfação dos pacientes em contexto hospitalar. Revista Gaúcha de Enfermagem; 37(2). Doi:10.1590/19831447.2016.02.55726.

Poghosyan, L., Shang, J., Liu, J., Poghosyan, H., Liu, N. \& Berkowitz, B. (2015). Nurse practitioners as primary care providers: Creating favorable practice environments in New York State and Massachusetts. Health Care Management Review, 40(1): 46-55. Doi:10.1097/HMR.0000000000000010. 
Stalpers, D., Brouwer, B. J. M., Kaljouw, M. J., \& Schuurmans, M. J. (2015). Associations between characteristics of the nurse work environment and five nurse-sensitive patient outcomes in hospitals: A systematic review of literature. International Journal of Nursing Studies, 52(4), 817-835. Doi:10.1016/j.jnurstu.2015.01.005.

Tomaszewska, K., Kłos, A., \& Majchrowicz, B. (2017). Influence of work environment on the quality of benefits provided by primary health care nurses. Journal of Education, Health and Sport, 7(8): 1191-1205. Doi:10.5281/zenodo.1039369.

Ventura, I., Ramalhal, T., \& Lucas, P. B. (2019). The nursing practice environment and nurses' satisfaction in the obstetrics hospital context: a scoping review. Annals of Medicine, 51(1), 203-203. Doi:10.1080/07853890.2018.1560162.

Weber, E., Ward, J. \& Walsh, T. (2015). Nurse leader competencies: A toolkit for success. Nursing Management, 46(12), 47-50. Doi:10.1097/01.NUMA.0000473505.23431.85.

Whittemore, R., \& Knafl, K. (2005). The integrative review: Updated methodology. Journal of Advanced Nursing, 52(5), 546-553. DOI: 10.1111/j.1365-2648.2005.03621.x.

Xian, M., Zhai, H., Xiong, Y., \& Han, Y. (2020). The role of work resources between job demands and burnout in male nurses. Journal of Clinical Nursing, 29(3-4), 535-544. Doi:10.1111/jocn.15103.

Zhu, X., Zheng, J., Liu, K., \& You, L. (2019). Rationing of nursing care and its relationship with nurse staffing and patient outcomes: The mediation effect tested by structural equation modeling. International Journal of Environmental Research and Public Health, 16(10). Doi:10.3390/ijerph16101672. 